Association for Information Systems

AIS Electronic Library (AISeL)

Wirtschaftsinformatik 2021 Proceedings

Track 18: Future of Digital Markets and

Platforms

\title{
A Systematic Literature Review of Digital Platform Business Models
}

Dennis Mallon

Technische Universität Berlin

Follow this and additional works at: https://aisel.aisnet.org/wi2021

Mallon, Dennis, "A Systematic Literature Review of Digital Platform Business Models" (2021).

Wirtschaftsinformatik 2021 Proceedings. 4.

https://aisel.aisnet.org/wi2021/GFuture18/Track18/4

This material is brought to you by the Wirtschaftsinformatik at AIS Electronic Library (AISeL). It has been accepted for inclusion in Wirtschaftsinformatik 2021 Proceedings by an authorized administrator of AIS Electronic Library (AISeL). For more information, please contact elibrary@aisnet.org. 


\title{
A Systematic Literature Review of Digital Platform Business Models
}

\author{
Dennis Mallon \\ Berlin Institute of Technology, Chair for Information and Communication Management, \\ Berlin, Germany \\ dennis.mallon@campus.tu-berlin.de
}

\begin{abstract}
Platforms and business models have been a subject of academic analysis and practical application for years. As digital platforms are significantly different due to an intervened and complex nature, typologies, fundamental concepts, and business models have been studied from separated perspectives. This paper reviews the platform and business model literature using a systematic literature review that identifies concepts underlying digital platforms. Henceforward, this research develops a working definition and links 109 business model components to 24 digital platform concepts to figure out what components constitute digital platforms' business models. Furthermore, the analysis shows that several digital platform concepts were deficient or not represented by business model components indicating the need for future research. The study concludes and discusses theoretical and practical implications, suggests future research areas, and marks its limitations.
\end{abstract}

Keywords: digital platform, business models, business model components

\section{Introduction}

Digital platforms, as drivers for our time's technical infrastructure, change permanently the way people and socio-technical ecosystems communicate, socialize, interact, consume, and share with one another [1-4]. The emergence of these largescale and multi-sided digital platforms disrupts numerous industries, such as transportation, banking, and retailing, and continue to change the traditional intermediation between supply and demand in our markets [5]. At its core, digital platforms coordinate and mediate between heterogeneous actors around a product, a resource, a service, or a technology based on direct or indirect network effects. The generated dynamics achieve growth by innovative and highly scalable business models that break familiar processes, intervene in exchange value chains, and gain exclusive access to customers [6, 7]. Digital platforms are embedded into more extensive digital infrastructures and compete on all technical and non-technical architecture levels while generating causal dynamics with users, internal resources, technical systems, complementors, and physical assets [8]. This generativity produces ecosystems that create research objects which surpass traditional information systems in size and scope [9]. The distributed internal structure and its intertwined connection to its environment

16th International Conference on Wirtschaftsinformatik,

March 2021, Essen, Germany 
pose massive research challenges and grow the scope and diversity of scientific discourse rapidly $[1,10]$.

As a unit of analysis and modeling for businesses, the concept of Business Models started to get attention in the 1990s [11-13]. Many definitions and interpretations of the business model concept were formed, leading to an inconsistent and even ambiguous state of research [7, 12, 14-16]. For instance, Osterwalder defined a Business Model as: "...conceptual tool that contains a set of elements and their relationships and allows expressing the business logic of a specific firm" [17, p. 3]. Schweiger et al. built on the research of Osterwalder and stated that business model components represent the smallest element of a business model and can therefore be used to examine specific parts of a business model in detail separately, such as the revenue model or the governance structure [18]. Nevertheless, a digital platform business model differs from traditional business models. Different models can be applied for sellers, buyers, complementors, and partners on various technical and non-technical architecture levels simultaneously [19]. The need for an accurate understanding of the digital platform business model and its components as a unit of analysis increases as aggregates such as industries, profit pools, or markets are no longer the ultimate references $[5,20]$. Therefore, this research uses a systematic literature review methodology to answer the following research questions (RQ).

RQ: What components constitute the business model of digital platforms and relate to the digital platforms' underlying concepts?

First, this review presents the methodological approach used during this research in section two. Second, section three provides a theoretical background on digital platform business models and defines its term in a working definition. Third, this research identifies the underlying concepts the literature is currently referring to when corresponding to digital platforms and presents the findings in a concept matrix after Webster and Watson [21]. In this study, concepts can be understood as abstract ideas or general notions mentioned by other authors that summarize certain phenomena observed in digital platforms. Also, abstract description, classification of platform mechanisms, description of characteristics, and digital platforms' peculiarities are summarized under concepts. Fourth, after extracting business model components from the literature, this research links these components to the digital platforms' underlying concepts and presents its results in section four. Fifth, section five discusses the results, derives theoretical implications and practical implications, indicates avenues of research activities for the future, and points to this study's limitations.

\section{$2 \quad$ Research design and methodology}

The following research is based on a systematic literature review [22]. It seeks to uncover the sources relevant to the digital platform business model to contribute to the business model research stream's relevance and rigor, explaining how one research 
builds on what is already known [23, 24]. The following overview provides a summary of the literature research procedure.

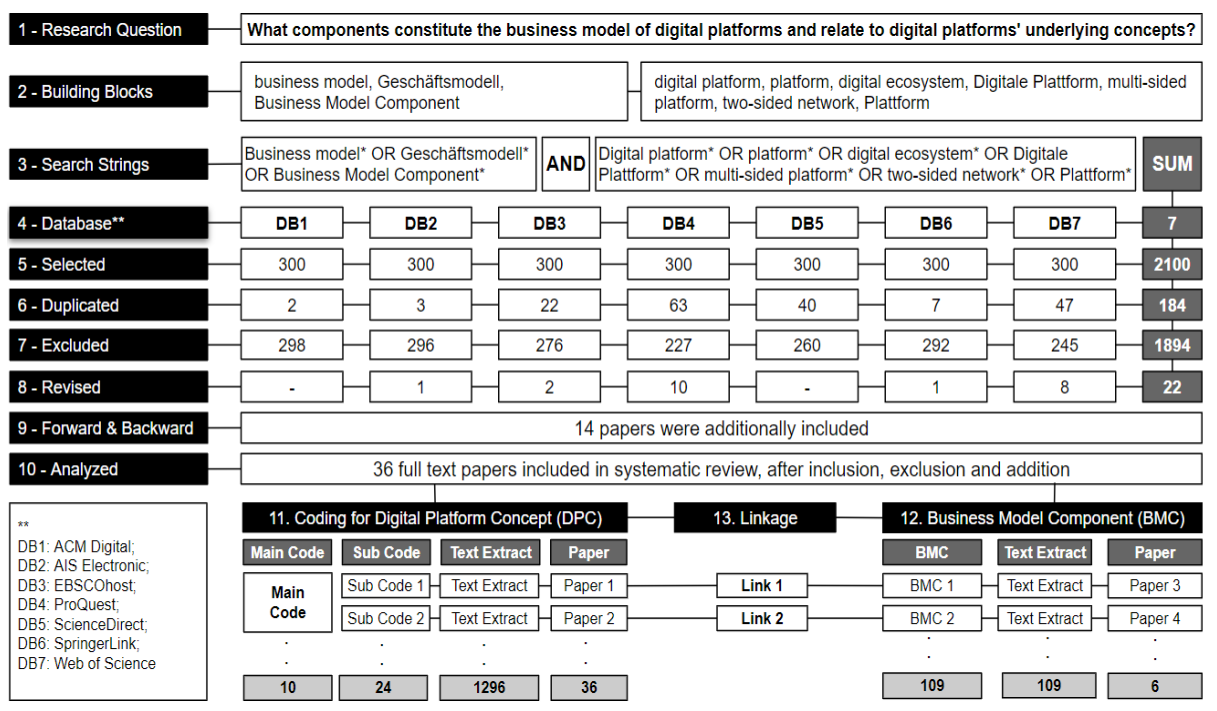

Figure 1. The systematic literature research process

The author divided the research question from section one into equivalent term fields, which are linked independently of one another, and then with one another [25]. This approach is called the block building method [25]. As a result, a so-called term matrix creates subject blocks and search terms according to a scheme illustrated in figure 1, steps two and three. The aim is to identify different synonyms for the subterms. Rowley and Slack also stated that it is commonly recommended to use a set of search phrases to exclude irrelevant contributions [24, 26]. Based on the subject blocks, the author derived and applied the following search strings: (digital platform* OR platform* OR digital ecosystem* OR Digitale Plattform* OR multi-sided platform* OR two-sided network* OR Plattform*) AND (Business model* OR Geschäftsmodell* OR Business Model Component*) to collect literature on the subsequent search library databases: ACM Digital Library, AIS Electronic, EBSCOhost, ProQuest, ScienceDirect, SpringerLink, Web of Science. For selecting the literature, the author used the database functionality to sort the results concerning the relevance of a return in the database. This study selected the highest-ranking records that appear at the top of the list based on the library database ranking system considering the database fields abstract, title, and keywords. A limitation was set to the first 300 papers per database due to the high return on hits. After this threshold, the author conducted a title and abstract screening but did not identify additional new concepts, which is a sign of near completion. A specific time range, as an example, the last five years, was not applied in this research as this limitation would not have included fundamental research. For instance, the concept of network effects, which has been significant for digital platforms, has been broadly discussed at the beginning of 2000. Also, articles in English 
and German were selected to reduces language bias. Roughly $2 \%$ of the article were written in German. The author screened the title, abstract, and keywords of 2100 articles, removed 184 duplicates, and subsequently applied the inclusion and exclusion criteria. Excluded from this research are studies about application development, benchmarks, crowdfunding, cybersecurity, education, farming, and political communication.

Furthermore, this research only included articles if a connection between digital platforms and business model components were indicated or if underlying digital platform concepts have been identified. Twenty-two articles were eligible for this review after this step. The author performed a forward-and-backward search and included additionally 14 articles [21, 24]. A backward search means going through the sources' bibliographies, and a forward search identifies articles that have cited the relevant publications, to include relevant literature [21]. This review analyzed 36 articles using an explorative coding process, which was repeated iteratively to develop conclusive coding constructs [27]. More specifically, 1296 text phrases have been extracted from the literature and iteratively coding into 24 digital platform subconcepts. For reasons of clarity, only concepts that were mentioned at least four times were considered. These sub-concepts were aggregated into ten digital platform concepts. Separately, this review screened the literature and extracted business model components, and further related these components to digital platform concepts. The business model components the author identified during the review of the literature, are given and used as a conceptual basis. The connection of business model components to digital platform concepts followed an iterative approach of linking a business model component by its description and definition. Henceforward, the results are presented and analyzed in section four.

\section{Theoretical background on digital platform business models}

Over the last couple of decades, there has been an extensive research on business models centering around how firms create, deliver, and capture value [28, 29]. Several literature reviews and investigations of the business model concept led to various scientific literature definitions and practical understandings [16]. Often cited in the literature are Zott et al., which define that: "A business model depicts the content, structure, and governance of transactions designed so as to create value through the exploitation of business opportunities" [30, p. 493]. Henceforward Teece defines: "A business model articulates the logic and provides data and other evidence that demonstrates how a business creates and delivers value to customers. It also outlines the architecture of revenues, costs, and profits associated with the business enterprise delivering that value" [31, p. 173].

With the emergence of digital technology and the ever-increasing importance, availability, and usability of data, traditional, analog, or offline business models get often disrupted [5]. For digital platforms does the digital technologies in use imply homogenization of data, editability, reprogrammability, distributedness, and selfreferentiality, which can lead to multiple inheritances in distributed settings, depending 
on the control mechanism and governance principles applied by the platform owner [4, $10,32]$. As all digital platforms build on a constantly evolving information technology, the digital infrastructure and its continually changing software base are vital drivers of dynamics and changes within the digital platform [3]. Therefore, Tiwana defines digital platforms to the extent that it: "...consists of an extensible codebase of a software-based system that provides core functionality shared by the modules that interoperate with it and the interfaces through which they interoperate" [33, p. 676]. As an extendible codebase enables third-party development of complementors via boundary resources, the integration of complementors is highly relevant for the digital platforms' design [34]. The boundary resource can exist on multiple digital platforms layers and often shift very rapidly [35]. Moreover, data as a boundary resource is gaining importance in practice. The users provide their data to the digital platform. The platform owner makes this data accessible via software tools, like Application Interfaces (API) and Software Development Kits (SDK) to complementors [1]. The platform and the complement often regulate this exchange by an arms' length relationships $[1,36]$. The integration is an incremental part of digital platforms. Multiple external parties, like users, providers of services, digital products, and complements, are invited on the layered, modular architecture to create value [37, 38]. Recently, Abdelkafi noted that a platform architecture is: "...a modularization that partitions the system into (1) a set of components whose design is stable and (2) a complementary set of components which are allowed - indeed encouraged - to vary" [39, p. 554]. Henceforward, the adaption to changes creates an incredibly complex task because organizations and business environments continuously evolve. The paradox of change implies the need for digital platforms to remain stable simultaneously and form a solid foundation for further enrolment and be sufficiently flexible to support unbounded growth and innovation effects [1, 40-42]. This digital platform's behavior is necessary to obtain the generativity, which describes the: "...overall capacity to produce unprompted changes driven by large, varied, and uncoordinated audiences" [43, p. 1980]. Several researchers stated the importance of the right balance between central and decentral structures because the governance determines whether the layered, modular architecture will successfully lever the innovation [33, 38, 44, 45]. Tiwana defines governance regarding who decides what and stated that: "...architecture can reduce structural complexity, governance can reduce behavioral complexity..." [46, p. 118]. Based on the work of Wareham et al., Constantinides describes the development of platform governance as a challenge, as it is how: "...to establish governance mechanisms that appropriately bound participant behavior without excessively constraining the desired level of generativity..." [38, 47, pp. 1195-1196]. The decision about openness and control mechanism applies on various levels, ranging from open interfaces to open source as bounding participants affect value creation and capture [1, 39, 48]. Therefore, in a closed platform, the fear of losing control of the platform owner can keep industry players from joining in the first place [39]. More users can be attracted in an open platform, creating a greater pool of potential contributors, which can lead to more innovations, probably in a shorter time frame [39, 49].

Further research adds to the technical understanding and characterizes digital platforms as a socio-technical assemblage encompassing the technical elements and 
associated organizational processes and standards [40]. The organization is primarily incremental for digital platforms as the entire culture, like mental models, skills, experiences, traditions, and the organizational identity, needs to relate to the digital setup, the underlying concepts, and its dynamics [50]. Moreover, the organizational set up needs to absorb the architectural modularity, as the organization needs to provide the variety and flexibility to handle technological trajectories [51]. Furthermore, it is essential to add non-technical aspects of digital platforms, such as the utilization as a mediator between different actors to facilitate the multi-party exchange of goods, services, or information to create value between the user and capture the value [20]. At its core, digital platforms enable a sharing system among user groups, providing digital services to communicate, conduct transactions, collect, process, and share data related to their common interests or activities [50]. Balancing the quality and quantity of the exchange enables a repeatable user interaction that is often facilitated in consumers' online communities [1,51]. Necessary for the transaction on platforms is the user's trust as it influences the platform's sales [52,53]. Schreieck et al. found that most digital platforms use a rating or review system to establish trust and to decrease perceived risk as users are more likely to use the platform due to the protective mechanisms [52]. These platforms are often categorized as marketplaces or transaction platforms and are subsumed under the definition of digital platforms for this research [34].

Centrally significant for digital platforms are network externalities or network effects as an enabler of dynamics to increase the single participants' utility as the platform's size grows [50]. Network effects can either be direct or indirect [7]. Network effects are direct, if the value of a digital platform depends on the number of users in the same user group, meaning it becomes more attractive for users as the total number of users on the same side increases $[1,11]$. Indirect network effects occur when the platform's value depends on the users' number in a different user group. It becomes more attractive for one group of users as the number of another group increases [11].

Additionally, digital platforms can apply economies of scale, meaning that the average cost declines as users' number increases [11]. The concept is not unique to digital platforms, but the effects are more evident as the marginal costs are often close to zero. The integration of user and complementors, initiate a constant innovation funnel whereby potential perspectives or ideas for innovations can be included, creating user acceptance $[14,54,55]$. Transactions, network effects, technical and non-technical adaption created various dynamics for digital platforms. Just recently, Abdelkafi et al. have shown that platform businesses' dynamics have been studied from three perspectives, the dynamics effects of digital platforms on markets and industry, the evolutionary dynamics of a platform, and competition effects among platforms [39]. The literature constitutes several delimiting and overlapping concepts and definitions depending on the author's perspectives and investigation area. Guggenberger et al. suggest subsuming digital platform business models under the definition and as a subtype of digital business models [34]. Also, Guggenberger et al. and Reuver et al. argue for the need to determine the subject of investigation. Therefore this research outlines a working definition based on the literature found during this review. Digital Platform Business Models are a conceptual extension of business models that operate on a continually evolving digital infrastructure, creating value while enabling 
interactions between user groups in the ecosystem, based on network effects $[11,34$, 50]. The digital infrastructure and the continually changing and extensible codebase of the software-based systems provide core functionality that enables integration of multiple parties via boundary resources and fosters value creation $[3,33]$. The digital platform business model incorporates the organizational needs to provide the variety and flexibility to handle technological trajectories to absorb the architectural modularity [51]. Digital platforms compete on all technical and non-technical architecture levels while generating causal dynamics and innovation funnels with users, internal resources, technical systems, complementors, and physical assets [8]. Overall, the focus lies on delivering digital offerings and digital experiences to customers building highly scalable business solutions in a socio-technical ecosystem [34, 39].

\section{$4 \quad$ Results}

This research identified 109 business model components found in the literature and linked them to 24 underlying digital platform concepts to answer the research question from section one. Figure 2 provides an overview of the results based on Webster and Watson [21].

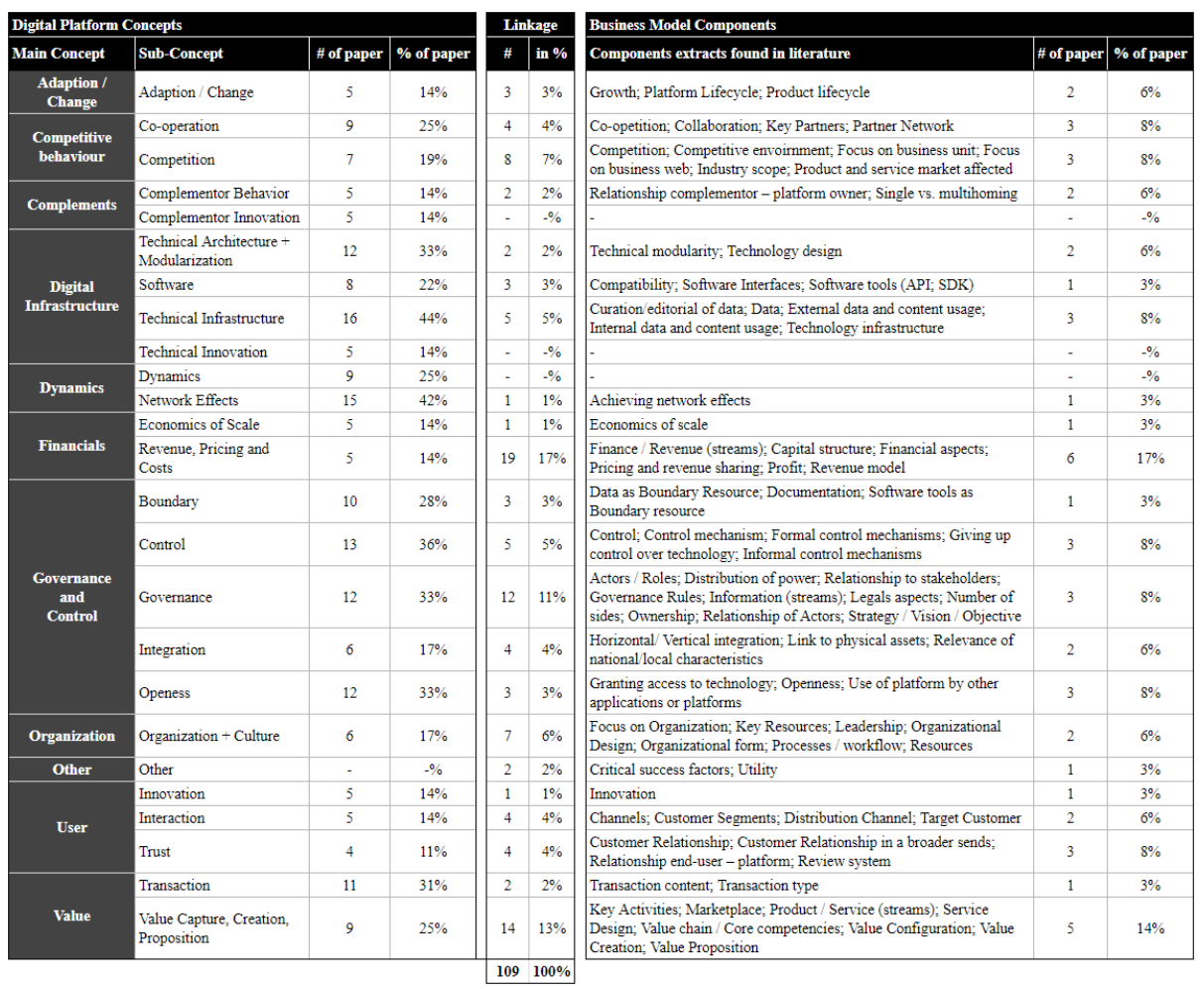

Figure 2. Matrix - business models components linked to digital platform concepts 
The matrix above aggregates the extracted and coded literature into main and subconcepts. The illustration shows the count of papers mentioned for the respective digital platform concept, in absolute and relative figures to the papers' total count. The listed business model components were linked according to the digital platform sub-concepts' alphabetical order. The count of papers mentioning business model components is shown in absolute and relative figures. Components with the same meaning are summarized in this matrix but are counted as occurred. The illustration shows the number of business model components linked to the digital platform sub concept under Linkage's headline. Afterward, the outcomes were indexed into a) the relevance of digital platform concept and b) the relevance of business model component towards digital platform concept. Furthermore, index a was subtracted from index b to determine the distance $\mathrm{c}$, as shown in the following formula.

$$
\text { Index } a-\text { Index } b=\text { Distance } c
$$

Henceforward the distances between $\mathrm{a}$ and $\mathrm{b}$ were categorized into $\mathrm{HR}$ - high representation, MR - medium/equal representation, LR - Low representation, VLR very low representation, NR - no representation. The results of the indexation are presented in figure 3 .

\begin{tabular}{|c|c|c|c|c|c|}
\hline \multicolumn{2}{|c|}{ Digital Platform Concept } & \multirow{2}{*}{ Index a } & \multirow{2}{*}{ Index b } & \multirow{2}{*}{ Distance c } & \multirow{2}{*}{ Category } \\
\hline Main Concept & Sub Concept & & & & \\
\hline Adaption / Change & Adaption/ Change & 0.31 & 0.16 & 0.15 & MR \\
\hline \multirow{2}{*}{$\begin{array}{l}\text { Competitive } \\
\text { behaviour }\end{array}$} & Co-operation & 0.56 & 0.21 & 0.35 & LR \\
\hline & Competition & 0.44 & 0.42 & 0.02 & MR \\
\hline \multirow{2}{*}{ Complements } & Complementor Behavior & 0.31 & 0.11 & 0.21 & MR \\
\hline & Complementor Innovation & 0.31 & 0.00 & 0.31 & NR \\
\hline \multirow{4}{*}{$\begin{array}{c}\text { Digital } \\
\text { Infrastructure }\end{array}$} & Software & 0.50 & 0.16 & 0.34 & LR \\
\hline & Technical Architecture + Modularization & 0.75 & 0.11 & 0.64 & VLR \\
\hline & Technical Infrastructure & 1.00 & 0.26 & 0.74 & VLR \\
\hline & Technical Innovation & 0.31 & 0.00 & 0.31 & $\mathrm{NR}$ \\
\hline \multirow{2}{*}{ Dynamics } & Dynamics & 0.56 & 0.00 & 0.56 & NR \\
\hline & Network Effects & 0.94 & 0.05 & 0.88 & VLR \\
\hline \multirow{2}{*}{ Financials } & Economics of Scale & 0.31 & 0.05 & 0.26 & LR \\
\hline & Revenue, Pricing and Costs & 0.31 & 1.00 & -0.69 & HR \\
\hline \multirow{5}{*}{$\begin{array}{l}\text { Governance } \\
\text { and } \\
\text { Control }\end{array}$} & Boundary & 0.63 & 0.16 & 0.47 & LR \\
\hline & Control & 0.81 & 0.26 & 0.55 & LR \\
\hline & Governance & 0.75 & 0.63 & 0.12 & MR \\
\hline & Integration & 0.38 & 0.21 & 0.16 & MR \\
\hline & Openess & 0.75 & 0.16 & 0.59 & VLR \\
\hline Organization & Organization + Culture & 0.38 & 0.37 & 0.01 & MR \\
\hline Other & Other & 0.00 & 0.11 & -0.11 & - \\
\hline \multirow{3}{*}{ User } & Innovation & 0.31 & 0.05 & 0.26 & LR \\
\hline & Interaction & 0.31 & 0.21 & 0.10 & MR \\
\hline & Trust & 0.25 & 0.21 & 0.04 & MR \\
\hline \multirow{2}{*}{ Value } & Transaction & 0.69 & 0.11 & 0.58 & VLR \\
\hline & Value Capture, Creation, Proposition & 0.56 & 0.74 & -0.17 & $\mathrm{HR}$ \\
\hline
\end{tabular}

Figure 3. Distance between the relevance of business model components in digital platforms 
Business model components linking to the concept of Revenue, Pricing, and Costs (distance: -0.69) and the concept of Value Creation, Value Capturing, and Value Proposition (-0.17) show a high representation as well as a distance below zero. The distance below zero indicates an overrepresentation or a lower relevance of these business model components for digital platform business models than other components, like the technical infrastructure. A high representation results from an intense investigation in the literature $[14,18,56]$.

The second category describes a distance between 0-0.2 and determines an equal or medium representation of the business model components toward the relevance as a digital platform concept. In this category, business model components linking to Adaption / Change (0.15), Competition (0.02), Complementor Behavior (0.21), Governance (0.12), Integration (0.16), Interaction (0.10), Organization + Culture (0.01) and Trust (0.04) showing a similar representation with its relevance to constitute the business model of digital platforms. Furthermore, an overlap of the description of business model components and digital platform concepts was identified [36, 56, 57].

A third category shows business model components with a distance between 0.2-0.5 towards the digital platform concepts. For instance, Boundary $(0.47)$ as a digital platform concept was mentioned in ten articles indicating a relatively high relevance for digital platforms. Also, research on digital platforms has emphasized the need to focus on boundaries between digital platforms and their ecosystem, where independent actors pragmatically engage innovations utilizing the opportunities and limitations of the digital or layered-modular architecture $[3,4,58]$. While investigating the boundary as a business model component, a lower relevance than other components indicates a low consideration of this concept in business model components. Similar to the subconcept Boundary, Co-operation (0.35), Control (0.55), Economics of Scale (0.26), Innovation (0.26), and Software (0.34) stipulate a relative underrepresentation toward other business model components, like the value creation.

As a fourth category, this research identified that concepts, specifically related to digital platforms, find a deficient representation in business model components. For instance, the concept of Network Effects (0.88), highly relevant as a fundamental concept for digital platforms' existence and operation, was linked to one business model component. Also, Openness (0.59), Technical Architecture + Modularization (0.64), Technical Infrastructure (0.74), Transaction (0.58) show a significantly lower representation as business model components than other components.

The fifth category presents digital platform concepts, where no business model components were relatable. Complementor Innovation (0.31), Dynamics (0.56), and Technical Innovation (0.31) found no consideration as a business model component. The category, Other, summarizes components like critical success factors and utility, which could not be related to platform concepts.

\section{$5 \quad$ Discussion and Conclusion}

This section concludes the theoretical and practical implications of this research, draws areas for future research based on the research findings, and states its limitations. 
Based on the findings, this research creates a working definition and relates 109 business model components to 24 digital platform concepts to figure out what components constitute digital platforms' business model. The results acknowledge that a strong research interest exists for business model components of non-digital platforms' as mainly technical and specifically platform concepts are relatively underrepresented by the components derived from the literature. A strong influence of economic and financial interest populates their relevance in the business model components. Also, Reuver et al. criticized the high interest in pricing strategies and financial dynamics rather than innovation dynamics within the economics literature [1].

\section{$5.1 \quad$ Theoretical Implications}

First, this review extends the definition of digital platform business models currently examined in the literature. It works on a more substantial connection of digital platform concepts and the business model research streams while integrating digital platform concepts to precisely define the subject of investigation. Therefore this research creates a working definition based on previous work and includes substantial aspects, like dynamics $[1,34]$. Still, the difficulty to clearly distinguish between digital and analog and the reflection in business models and components will be a challenging research subject as the digitalization extends the scope of information technology in almost all areas of these socio-technical ecosystems [1].

Second, this study extracts underlying digital platform concepts and presents its relevance currently discussed in the literature. By analyzing the results, areas of the current research interest have been identified. Pointing out the complexity and unique aspects such as network effects helps to understand how digital platforms take over large parts of markets across industries [5]. Furthermore, this study includes usercentric platform business model components such as the users' trust and interactions and therefore adds to prior literature.

Third, this study elaborates business model components constituting digital platform business models. By comparing the relevance of the digital platform concept to the relevance of business model components linked to these concepts, this research identifies five categories. The relative underrepresentation of the digital platform concept, like network effects, technical innovation, and the platform dynamics, indicates the need to further investigate the role of business model components and their adaption through digitalization in digital platform business models.

\subsection{Practical Implications}

First, this study contributes to the analysis of digital platforms. Without an investigation of the underlying concepts necessary for digital platforms, a holistic understanding of digital platforms and their generative existence is lacking. This study elaborates and derives these platform concepts from the literature, helping practices to design digital platform business model. For instance, this study contributes to increase the awareness for practice to consider an ecosystemic viewpoint and integrate the dynamics created in digital platforms' intertwined nature. Therefore, this research 
further points out the importance of anticipating changes, adjusting business models, and aligning complementarities to sustain platform viability [44, 59].

Second, this research contributes to the application of the business model concepts. As business model components represent the smallest element of a business model that examines specific parts of a business model in detail, this research analyzes these components' relevance in digital platform business models. This investigation helps practices to consider additional components relevant in applying business model concepts. Without an adaption to the emergence of digital change around business models, Osterwalder's concept of nine blocks probably can be getting less useable in practice increases the risk of a more defective application $[13,60]$.

\subsection{Future Research}

Digital platforms make a difference to existing concepts due to their digital infrastructure, the modularization, the integration of complements, the applied governance and controls, the evolving causal relationship within the ecosystem, the innovation dynamics, and the internalization based on network effects $[1,3]$. A clear distinction between business model components gets less accurate due to the emergence of information technology, like automation, machine learning, and artificial intelligence $[1,3,39]$. The need increases to anticipate the influence of digitalization and its effects on the business model components itself. This research proposes in Research Area 1 that further research efforts enhance the business model concept holistically and include dynamics, the innovation, digitally, by users and complementors. Furthermore, it would be worth investigating other business model components, like the user interaction and their adaption to constant digital platforms changes.

Digital platforms use their technical architectures and organizational structures as a source of strategic opportunity to change their directions and relationships over time [39]. These underlying causal relationship should be known and govern carefully [38]. The integration into the associated ecosystems and, in turn, to other ecosystems increases the risk of unforeseen effects in case of unexpected and no manageable changes $[53,59]$. Most platforms use the data gathered from transactions and enhance the causal grid as briefly described in the following: more users generate more data, which can be used to improve user experience, which attracts more users because the platform has more users and more data, it can deliver better advertisement campaigns and thereby attract more revenues, which in turn can be used to improve user experience, which attracts more users [11, 61]. This research proposes in Research Area 2 to investigate digital platforms' causalities using an appropriate modeling language to enhance the mental model of decision-makers, users, complementors, and regulators $[62,63]$.

Furthermore, the digital platform replaces horizontal and vertical structures with an ecosystemic understanding. Most business model concepts to date still overlook the systemic participation of actors $[5,11,64]$. Digital platforms bring together multiple user groups on various levels of their architecture and create network externalities. This intersection between users, complementors regulators, and digital platforms requires a 
systematical understanding $[1,3,35]$. For instance, Beer compared business systems to biological systems, emphasizing that organizations as an organism respond to their environment [65]. This ecological perspective argues that the market economy is best understood as a living evolving ecosystem $[7,65,66]$. This research proposes in Research Area 3 to increase the effort to analyze digital platforms from a system thinking viewpoint, applying system models to emphasize the impact on the sociotechnical ecosystem we humans also belong.

\section{$5.4 \quad$ Limitations}

This study's limitation lies in the fact that this research was done by one reviewer, which implies a high researcher bias of applying inclusion and exclusion criteria. A structured approach, including a reiterated critical reflection on the decisions, has been chosen to reduce individual bias by the author. Nevertheless, a second and a third researcher would have been provided more objectivity. Additionally, the high amount of hits returned by one literature database opens the questions of this literature database's request. Also, the proposed systematic procedure was enhanced iteratively. During the research process, several studies were added due to the researcher's decision.

\section{Acknowledgments}

The author is grateful and wishes to thank the anonymous reviewers for their constructive comments. The Editors-in-Chief's significant encouragement and help in providing excellent feedback and advice are much appreciated and helped to improve the overall quality of this research endeavor.

\section{References}

1. Reuver, M. de, Sørensen, C., Basole, R.C.: The Digital Platform: A Research Agenda. Journal of Information Technology 33, 124-135 (2018)

2. Hein, A., Scheiber, M., Böhm, M., and Weking, J.: Toward a Design Framework for Service-Platform Ecosystems, Research Papers. 132 (2018)

3. Hein, A., Schreieck, M., Wiesche, M., Böhm, M., Krcmar, H.: Digital platform ecosystems. Electron Markets 30, 87-98 (2020)

4. Yoo, Y., Henfridsson, O., Lyytinen, K.: The New Organizing Logic of Digital Innovation: An Agenda for Information Systems Research. Information Systems Research 21, 724735 (2010)

5. Demil, B., Lecocq, X., \& Warnier, V.: "Business model thinking", business ecosystems and platforms: the new perspective on the environment of the organization. M@n@gement, 21(4), 1213-1228 (2018)

6. Bundesministerium für Wirtschaft und Energie: Weissbuch-Digitale Plattformen. Digitale Ordnungspolitik für Wachstum, Innovation, Wettbewerb und Teilhabe (2017)

7. Kim, J.: The platform business model and business ecosystem: quality management and revenue structures. Manchester (2016) 
8. Pon, B., Seppälä, T., Kenney, M.: Android and the demise of operating system-based power: Firm strategy and platform control in the post-PC world. Telecommunications Policy 38, 979-991 (2014)

9. Sørensen, C., Landau, J.S.: Academic agility in digital innovation research: The case of mobile ICT publications within information systems 2000-2014. The Journal of Strategic Information Systems 24, 158-170 (2015)

10. Henfridsson, O., Mathiassen, L., Svahn, F.: Managing Technological Change in the Digital Age: The Role of Architectural Frames. Journal of Information Technology 29, 27-43 (2014)

11. Fehrer, J.A., Woratschek, H., Brodie, R.J.: A systemic logic for platform business models. Journal of Service Management 29, 546-568 (2018)

12. Amit, R.H., Zott, C.: Business Model Innovation: Creating Value in Times of Change. Creating Value in Times of Change. SSRN Journal (2010)

13. Osterwalder, A., Pigneur, Y., Tucci, C.L.: Clarifying Business Models: Origins, Present, and Future of the Concept. Communications of the Association for Information Systems $16,1-25$ (2005)

14. Krcmar, H., Friesike, S., Bohm, M., Schildhauer, T.: Innovation, Society and Business: Internet-Based Business Models and Their Implications. SSRN Journal (2012)

15. Zott, C., Amit, R., Massa, L.: The Business Model: Recent Developments and Future Research. Journal of Management 37, 1019-1042 (2011)

16. Teece, D.J.: Business models and dynamic capabilities. Long Range Planning 51, 1, 40-49 (2018)

17. Yablonsky, S.: A Multidimensional Framework for Digital Platform Innovation and Management: From Business to Technological Platforms. Systems Research and Behavioral Science 35, 485-501 (2018)

18. Schweiger, A., Nagel, J., Böhm, M., Krcmar, H.: Platform Business Models. In: Project Consortium TUM Living Lab Connected Mobility (ed.) Digital Mobility Platforms and Ecosystems, pp. 66-77. mediaTUM (2016)

19. Täuscher, K.: Business Models in the Digital Economy: An Empirical Classification of Digital Marketplaces

20. Sorri, K., Seppänen, M., Still, K., \& Valkokari, K.: Business Model Innovation with Platform Canvas. Journal of Business Models, 7(2), 1-13 (2019)

21. Webster, J., Watson, R.T.: Analyzing the Past to Prepare for the Future: Writing a Literature Review. Management Information Systems Quarterly 26, xiii-xxiii (2002)

22. Paré, G., Trudel, M.-C., Jaana, M., Kitsiou, S.: Synthesizing information systems knowledge: A typology of literature reviews. Information \& Management 52, 183-199 (2015)

23. Shaw, J.: A schema approach to the formal literature review in engineering theses. System 23, 325-335 (1995)

24. Vom Brocke, J., Simons, A., Niehaves, B., Niehaves, B., Riemer, K., Plattfaut, Ralf \& Cleven, Anne: Reconstructing the Giant: On the Importance of Rigour in Documenting the Literature Search Process, 2206-2217

25. Guba, B.: Systematische Literatursuche. Wiener medizinische Wochenschrift 158, 62-69 (2008)

26. Rowley, J., Slack, F.: Conducting a literature review. Management Research News 27, 3139 (2004)

27. Saldaña, J.: The coding manual for qualitative researchers. SAGE, Los Angeles, Calif., London, New Delhi, Singapore, Washington DC (2016) 
28. Foss, N.J., Saebi, T.: Fifteen Years of Research on Business Model Innovation. Journal of Management 43, 200-227 (2017)

29. Župič, I., Budler, M., Trkman, P.: Characterization of Business Model Research. Bibliometric Analysis and the Future Agenda. In: Digital Transformation - From Connecting Things to Transforming Our Lives, pp. 719-731. University of Maribor Press (2017)

30. Amit, R., Zott, C.: Value creation in E-business. Strategic Management Journal 22, 493 $520(2001)$

31. Teece, D.J.: Business Models, Business Strategy and Innovation. Long Range Planning 43, 172-194 (2010)

32. Kallinikos, J., Aaltonen, A., Marton, A.: The Ambivalent Ontology of Digital Artifacts. Management Information Systems Quarterly 37, 357-370 (2013)

33. Tiwana, A., Konsynski, B., Bush, A.A.: Platform Evolution: Coevolution of Platform Architecture, Governance, and Environmental Dynamics. Information Systems Research 21, 675-687 (2010)

34. Guggenberger, T., Möller, F., Boualouch, K., and Otto, B.: Towards a Unifying Understanding of Digital Business Models (2020)

35. Walton, N.: Ecosystems Thinking and Modern Platform-Based Ecosystem Theory. In: Walton, N. (ed.) The Internet as a Technology-Based Ecosystem, 17, pp. 85-117. Palgrave Macmillan UK, London (2017)

36. Schreieck, M., Wiesche, M., Krcmar, H.: Design and Governance of Platform Ecosystems. - Key Concepts and Issues for Future Research. ECIS 2016 Proceedings Research Papers, 76. (2016)

37. Foerderer, J., Kude, T., Mithas, S., Heinzl, A.: Does Platform Owner's Entry Crowd Out Innovation? Evidence from Google Photos. Information Systems Research 29, 444-460 (2018)

38. Constantinides, P., Henfridsson, O., Parker, G.G.: Introduction-Platforms and Infrastructures in the Digital Age. Information Systems Research 29, 381-400 (2018)

39. Abdelkafi, N., Raasch, C., Roth, A., Srinivasan, R.: Multi-sided platforms. Electron Markets 29, 553-559 (2019)

40. Tilson, D., Sorensen, C., Lyytinen, K.: Change and Control Paradoxes in Mobile Infrastructure Innovation: The Android and iOS Mobile Operating Systems Cases. In: 2012 45th Hawaii International Conference on System Sciences, pp. 1324-1333. IEEE (2012)

41. Tilson, D., Lyytinen, K., Sørensen, C.: Digital Infrastructures: The Missing IS Research Agenda. Information Systems Research 21, 748-759 (2010)

42. Resca, A., Za, S., Spagnoletti, P.: Digital Platforms as Sources for Organizational and Strategic Transformation: A Case Study of the Midblue Project. J. theor. appl. electron. commer. res. 8, 11-12 (2013)

43. Zittrain, J.: The Generative Internet. Harvard Law Review 52, 1974 (2006)

44. Parker, G., van Alstyne, M., Jiang, X.: Platform Ecosystems: How Developers Invert the Firm. How developers invert the firm. Management Information Systems Quarterly 41, 255-266 (2017)

45. Rochet, J.-C., Tirole, J.: Platform Competition in Two-Sided Markets. Journal of the European Economic Association 1, 990-1029 (2003)

46. Tiwana, A.: Platform ecosystems. Aligning architecture, governance, and strategy. Morgan Kaufmann, Waltham, MA (2013)

47. Wareham, J.D., Fox, P.B., Cano Giner, J.L.: Technology Ecosystem Governance. Organization Science, 1195-1215 (2013) 
48. Eisenmann, T.R.: Managing Proprietary and Shared Platforms. California Management Review 50, 31-53 (2008)

49. Huber, T.L., Kude, T., Dibbern, J.: Governance Practices in Platform Ecosystems: Navigating Tensions Between Cocreated Value and Governance Costs. Information Systems Research 28, 563-584 (2017)

50. Eferin, Y., Hohlov, Y., Rossotto, C.: Digital platforms in Russia: competition between national and foreign multi-sided platforms stimulates growth and innovation. Digital Policy, Regulation and Governance 21, 129-145 (2019)

51. Spagnoletti, P., Resca, A., Lee, G.: A Design Theory for Digital Platforms Supporting Online Communities: A Multiple Case Study. Journal of Information Technology 30, 364380 (2015)

52. Schreieck, M., Hein, A., Wiesche, M., Krcmar, H.: The Challenge of Governing Digital Platform Ecosystems. In: Linnhoff-Popien, C., Schneider, R., Zaddach, M. (eds.) Digital Marketplaces Unleashed, 21, pp. 527-538. Springer Berlin Heidelberg, Berlin, Heidelberg (2018)

53. Yun, J.J., Won, D., Park, K., Yang, J., Zhao, X.: Growth of a platform business model as an entrepreneurial ecosystem and its effects on regional development. European Planning Studies 25, 805-826 (2017)

54. Chesbrough, H.: Business Model Innovation: Opportunities and Barriers. Long Range Planning 43, 354-363 (2010)

55. Surowiecki, J.: The Wisdom of Crowds. Why the many are smarter than the few and how collective wisdom shapes business, economies, societies, and nations. Knopf Doubleday Publishing Group, New York (2005)

56. Nooren, P., van Gorp, N., van Eijk, N., Fathaigh, R.Ó.: Should We Regulate Digital Platforms? A New Framework for Evaluating Policy Options. Policy \& Internet 10, 264 301 (2018)

57. Täuscher, K., Laudien, S.M.: Understanding platform business models: A mixed methods study of marketplaces. European Management Journal 36, 319-329 (2018)

58. Eaton, B., Elaluf-Calderwood, S., Sørensen, C., Yoo, Y.: Distributed Tuning of Boundary Resources: The Case of Apple's iOS Service System. Management Information Systems Quarterly 39, 217-243 (2015)

59. Teece, D.J.: Dynamic capabilities and (digital) platform lifecycles. Advances in Strategic Management, 37, 211-225 (2017)

60. Groesser, S.N., Jovy, N.: Business model analysis using computational modeling: a strategy tool for exploration and decision-making. Journal of Management Control 27, 6188 (2016)

61. Prüfer, J., \& Schottmuller, C.: Competing with Big Data CentER Discussion Paper. SSRN Journal (2017)

62. Forrester, J.W.: Industrial dynamics. Martino Publ, Mansfield Centre, Conn. (2013)

63. Groesser, S.N., Schaffernicht, M.: Mental models of dynamic systems: taking stock and looking ahead. System Dynamics Review 28, 46-68 (2012)

64. Wieland, H., Hartmann, N.N., Vargo, S.L.: Business models as service strategy. Journal of the Academy of Marketing Science 45, 925-943 (2017)

65. Beer, S.: Brain of the firm. John Wiley \& Sons, Chichester (1995)

66. Rothschild, M.L.: Bionomics. Economy as ecosystem. Holt, New York (1992) 